\title{
Video Article \\ In Vivo Protocol of Controlled Subconcussive Head Impacts for the Validation of Field Study Data
}

\author{
Zachary W. Bevilacqua ${ }^{1}$, Megan E. Huibregtse ${ }^{1}$, Keisuke Kawata ${ }^{1,2}$ \\ ${ }^{1}$ Department of Kinesiology, School of Public Health-Bloomington, Indiana University \\ ${ }^{2}$ Program in Neuroscience, College of Arts and Sciences, Indiana University
}

Correspondence to: Keisuke Kawata at kkawata@indiana.edu

URL: https://www.jove.com/video/59381

DOI: doi:10.3791/59381

Keywords: Behavior, Issue 146, Subconcussion, soccer heading, chronic traumatic encephalopathy, repeated measures, methodology, concussion

Date Published: 4/18/2019

Citation: Bevilacqua, Z.W., Huibregtse, M.E., Kawata, K. In Vivo Protocol of Controlled Subconcussive Head Impacts for the Validation of Field Study Data. J. Vis. Exp. (146), e59381, doi:10.3791/59381 (2019).

\section{Abstract}

Subconcussive hits pose a threat to neuronal health as they have shown to induce neuronal structural damage and functional impairment without causing outward symptomology and appear to be a key contributor to an irreversible neurodegenerative disease, chronic traumatic encephalopathy (CTE). In addition, athletes can incur more than 1,000 of these hits per season. The subconcussive soccer heading model (SSHM) is a relevant, reproducible, and leading method of isolating and examining the effects of these subconcussive head impacts. By controlling variables such as ball traveling speed, the frequency of impacts, interval, ball placement to the head, as well as by measuring head impact magnitude, the SSHM provides the scientific community with a superior avenue of investigating the acute subconcussive effects on neuronal health. In this paper, we demonstrate the utility of SSHM in studying a time-course expression of neurofilament-light polypeptide (NF-L) in plasma in a repeated measures fashion. NF-L is an axonal injury marker that has previously been shown to be elevated in boxers and football players following subconcussive head trauma. Thirty-four adult aged soccer players were recruited and randomly assigned to either a soccer heading $(n=18)$ or kicking $(n=16)$ group. The heading group executed 10 headers with soccer balls projected at a velocity of $25 \mathrm{mph}$ over 10 min. The kicking group followed the same protocol with 10 kicks. Plasma samples were obtained before and at $0 \mathrm{~h}, 2 \mathrm{~h}$, and $24 \mathrm{~h}$ after heading/ kicking and assessed for NF-L expressions. The heading group showed a gradual increase in plasma NF-L expression and peaked at $24 \mathrm{~h}$ after the heading protocol, whereas the kicking group remained consistent across the time points. These results confirmed the NF-L data from clinical field studies, encouraging the use of SSHM to validate clinical subconcussion data.

\section{Video Link}

The video component of this article can be found at https://www.jove.com/video/59381/

\section{Introduction}

Long-term, repetitive exposure to subconcussive head impacts has been proposed as one of the key contributors for developing the neurodegenerative disease CTE ${ }^{1,2,3,4,5}$. Each year, approximately 2.5 million high school and college athletes engage in contact sports that frequently induce these subconcussive insults through rapid acceleration-deceleration of the body and head ${ }^{6,7}$. Specifically, contact sport athletes may experience several 100 up to a 1,000 of such impacts per season ${ }^{6,8,9}$. Additionally, other populations, such as military men and women, have registered more than 300,000 head injuries since 2001, which has manifested into the recent diagnosis of CTE within a retired military veteran $^{10}$. This diagnosis parallels with 110 postmortem CTE brains of American football players and four postmortem soccer players to present a burgeoning public health issue ${ }^{11,12}$. In light of the staggering prevalence, head impact research must shift its gaze to incorporate sound, precise methods of analyzing the acute debt subconcussive hits are inducing in a variety of arenas.

The SSHM presented here is one that satisfies the current methodological need to safely induce common mechanical stresses placed upon neural tissue during contact sport activities. The implementation of this model allows investigators to meticulously manage ball traveling speed, the frequency of impacts, interval, ball placement to the head, as well as measurements of head impact magnitude ${ }^{13,14}$. While these factors are virtually impossible to control in the field setting, the SSHM provides an outlet for researchers to isolate the effects of subconcussive head impacts. Furthermore, through the elimination of confounding variables seen during play (e.g., effects from vigorous exercise, body damage, body temperature change, and hydration/perspiration), the SSHM provides a superior method of validating clinical observations.

The SSHM has direct parallels to head impacts seen specifically within the realm of sport. As such, the literature has already begun to show its utility and corroborate the findings of cumulative head impact burden from other investigators. For example, we have demonstrated that the burden of subconcussive head impacts significantly drive neuro-ophthalmologic dysfunction amongst soccer athletes ${ }^{13,15}$. In addition, as few as 10 subconcussive impacts have been shown to immediately perturb vestibular function which can be normalized after $24 \mathrm{~h}$ of resting ${ }^{16}$. In this methodological report, we describe the application of SSHM to safely study the effects of subconcussive head impacts and introduce one of our findings that repetitive subconcussive head impacts gradually increase the concentration of a neuron-derived blood biomarker, namely NF-L ${ }^{14}$. 
This finding not only substantiates previous results of NF-L presence due to repetitive subconcussive blows to the head ${ }^{17,18}$ but also validates that the SSHM can reproduce such findings in a controlled clinical manner.

\section{Protocol}

The authors verify that the Indiana University Institutional Review Board approved the study (protocol \# 1610743422) and written informed consent was obtained.

NOTE: The SSHM was integrated into a repeated measures design that is intended to investigate changes among dependent variables within subjects at $0 \mathrm{~h}, 2 \mathrm{~h}$, and $24 \mathrm{~h}$ post-intervention compared to individual pre-intervention values. This study design allows researchers to track changes for a $24 \mathrm{~h}$ period, which is the typical timeframe between athletic practices. In the present study, soccer players were randomly assigned to either a soccer heading $(n=18)$ or a soccer kicking group $(n=16)$.

\section{Setup}

1. Following baseline measurement collection (pre-intervention), begin the SSHM with the positioning of a soccer ball launcher approximately 40 $\mathrm{ft}$ away from the subject, as well as ensuring the soccer ball is inflated to $9 \mathrm{psi}$.

2. The face of the machine displays two identical dials, which regulate the speed of the left and right wheels, and an on/off switch in between them. Set both these dials to a standardized speed of choice.

NOTE: For this demonstration purpose, the ball traveling speed has been set to $30 \mathrm{mph}$. This speed was chosen as it simulates a soccer throw-in from the sideline to midfield. Soccer players frequently perform this maneuver during practice and games.

3. Place 3 inch blocks underneath the wheels of the ball launcher to allow for the desired trajectory (no blocks needed for kicking subjects). Once this is complete, the ball launcher is then angled to $40^{\circ}$, which is measured as the angle between the ground and the midline of the rotating wheels.

NOTE: The angle measurement is taken with a goniometer, and the machine can be adjusted after loosening a knob located along the blue rails where the soccer ball is loaded.

4. Once the soccer machine is properly set, fit the subjects with a triaxial accelerometer embedded within a head-band pocket and positioned directly below the external occipital protuberance (inion) to monitor linear and rotational head accelerations.

5. Start up the corresponding software for the accelerometer, and enter the subject's information accordingly. At this point, the subject is ready to begin the familiarization trials of the intervention (heading, kicking).

\section{Familiarization trials}

1. Position the subject approximately $40 \mathrm{ft}$ in front of the ball launcher.

2. Be sure to explain to the subject that the ball launcher will volley the soccer ball to them and that they simply need to simulate intervention contact with the ball (i.e., heading subjects will catch the ball with their hands in front of their forehead before head-to-ball contact is made, kicking subjects will "trap" the ball on the ground with their foot instead of volleying the ball back, and standing subjects will remain static and not make contact with the ball).

3. When the subject understands and feels ready, have the researcher turn the ball launcher on, load the soccer ball onto the blue rails, and finally push the ball into the rotating wheels after a 3-2-1 countdown.

4. After stopping the ball as described previously (step 2.2), have the subject roll the ball back.

5. Repeat steps 2.3 and 2.4 two to four additional times (no rest time required between) to ensure that the subject positioning is correct and interaction with the ball will be safe and controlled. This concludes the familiarization trials.

\section{Intervention}

1. Verbally confirm that the subject is ready. Once confirmed, give instructions to the heading subjects to only make forehead contact with the ball; tell the subjects to avoid potential impacts to the crown, parietal, and temporal lobes. Instruct the kicking subjects to kick the ball only while it is in flight as ball contact with the ground will attenuate the subsequent impact to the foot.

2. Instruct both heading and kicking subjects to volley the ball to a target (additional researcher) approximately half the distance between them and the machine. As best they can, ask subjects to do this in a manner that will mimic the arched trajectory the ball took during its flight toward the subject.

3. Activate the triaxial accelerometer and begin the recording.

4. Load the soccer ball onto the blue rails, push the ball into the rotating wheels after a 3-2-1 countdown, and make sure appropriate contact is made.

5. Repeat step 3.4 nine more times with a $60 \mathrm{~s}$ rest in between bouts. If the subject forgoes interaction with the ball (due to inopportune placement or suspected contact with the body in an area that must be avoided), then volley the ball to the subject again promptly, without any rest period.

6. In between each head contact with the ball, verify that the triaxial accelerometer registered an impact (using triaxial software; kicking subjects should not register G-force).

7. Once the intervention is concluded, turn off the ball launcher and stop the triaxial recording (important, as the movement required to remove the headband could record another "impact"). Once the recording has stopped, remove the headband. 


\section{Representative Results}

The results represented here were interpreted from a previous article ${ }^{14}$, in which the SSHM was utilized as previously described. In this particular study, we aimed to show how the SSHM could induce changes in plasma levels of NF-L, which is an axonal injury marker that is hypothesized to filter out of the cranium and into the peripheral blood following head impacts.

\section{SSHM and head kinematics}

The present data were derived from 34 subjects who were eligible for analysis (heading group: $n=18$, and kicking [control] group: $n=16$ ). There were no significant differences in any demographic characteristic between the groups. Demographics and head impact kinematics are detailed in Table 1. Head impact kinematic data showed that the heading group experienced a median linear head acceleration of $31.8 \mathrm{~g}$ per head impact (IQR: $31.1-34.5 \mathrm{~g}$ ) and a median rotational head acceleration of $3.56 \mathrm{krad} / \mathrm{s}^{2}$ per head impact (IQR: $2.93-4.04 \mathrm{krad} / \mathrm{s}^{2}$ ). In contrast, the kicking (control) group did not result in detectable levels of head acceleration (as expected) (Table 1).

\section{SSHM effects on NF-L biomarker levels}

The SSHM was able to produce the following outcomes. See Figure 1 for the visual representation of the results ${ }^{14}$. (i) There was a gradual increase in plasma NF-L expression, as illustrated by a statistically significant time effect for the head impact group, $\mathrm{F}(1,31)=9.17, p=0.0049$. For example, $0.03 \mathrm{pg} / \mathrm{mL}$ of NF-L is estimated to increase every hour after 10 headers (SE $=0.001$ ). (ii) There was no significant time effect for the kicking (control) group, $\mathrm{F}(1,31)=1.20, p=0.28$. (iii) Follow-up paired $t$-tests with Bonferroni correction within the heading group revealed that a significant difference appeared at $24 \mathrm{~h}$ post-heading $(3.68 \pm 0.30 \mathrm{pg} / \mathrm{mL})$ compared to pre-heading $(3.12 \pm 0.29 \mathrm{pg} / \mathrm{mL}, p=0.0013 ; \mathrm{Cohen}$ 's $\mathrm{d}=1.898$ ). (iv) Linear regression, adjusting for the baseline NF-L level, was used to assess the between-group difference at the $24 \mathrm{~h}$ postintervention time point and distinguished that the NF-L level in the heading group was significantly higher than the kicking (control) group with an estimated mean difference of $0.66 \mathrm{pg} / \mathrm{mL}(\mathrm{SE}=0.22, p=0.0025)$.

\begin{tabular}{|l|l|l|l|}
\hline Variables & Heading & Kicking Control & P-value \\
\hline $\mathrm{n}$ & 18 & 16 & - \\
\hline $\mathrm{Sex}$ & $7 \mathrm{M} 11 \mathrm{~F}$ & $6 \mathrm{M} 10 \mathrm{~F}$ & - \\
\hline Age, $\mathrm{y}$ & $20.3 \pm 1.5$ & $21.2 \pm 1.4$ & 0.089 \\
\hline BMI, $\mathrm{kg} / \mathrm{m}^{2}$ & $23.2 \pm 2.4$ & $24.4 \pm 3.2$ & 0.236 \\
\hline No. of previous concussion & $0.78 \pm 1.0$ & $0.63 \pm 1.7$ & 0.753 \\
\hline Soccer heading experience, $\mathrm{y}$ & $9.5 \pm 3.6$ & $10.0 \pm 4.5$ & 0.725 \\
\hline $\begin{array}{l}\text { Head impact kinematics, median } \\
\text { IIQR), a }\end{array}$ & & & \\
\hline PLA, g & $31.8(31.1-34.5)$ & - \\
\hline PRA, krad/s ${ }^{2}$ & $3.56(2.93-4.04)$ & - \\
\hline $\begin{array}{l}\text { Note: BMI, body mass index. IQR, interquartile range. PLA, peak linear acceleration. PRA, peak rotational acceleration. krad, kiloradian. } a, \text { Based } \\
\text { on the sum of 10 soccer headers. b, Soccer kicking did not cause a detectable level of head acceleration. }\end{array}$ \\
\hline
\end{tabular}

Table 1: Demographics and impact kinematics by group. 


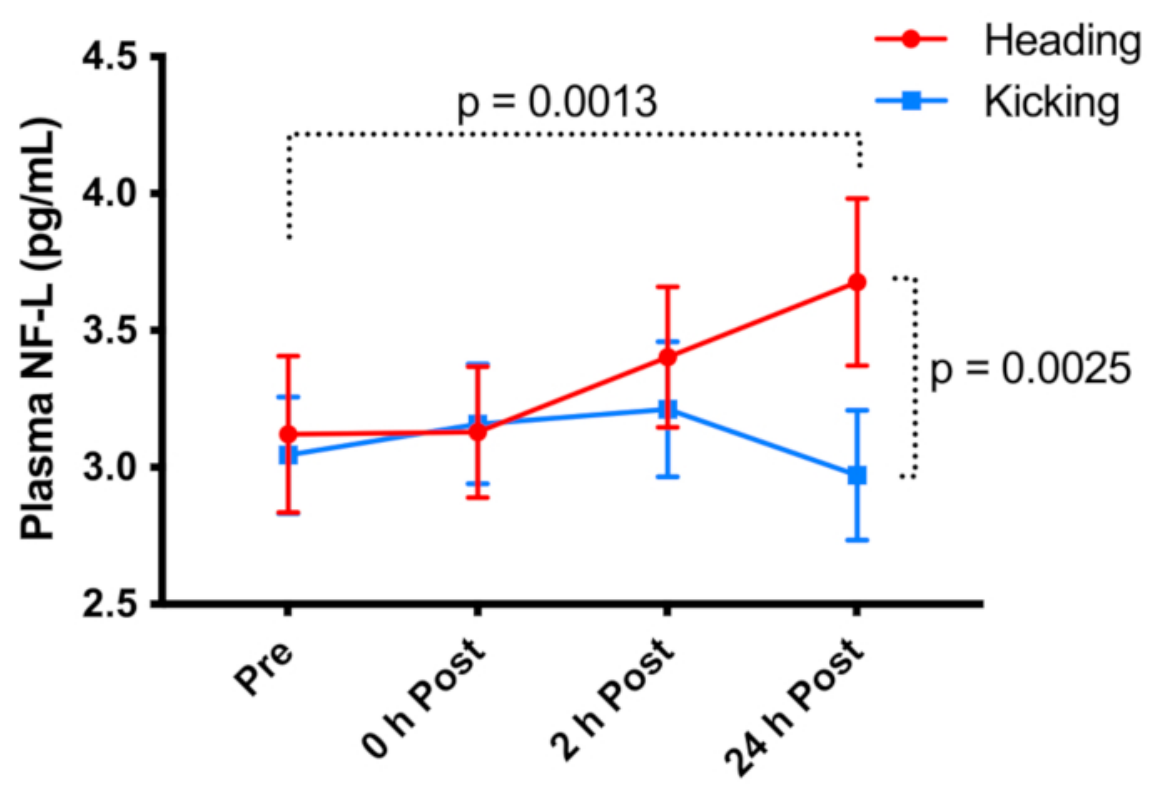

Figure 1: Changes in plasma NF-L levels before and after subconcussive impacts. In the heading group, NF-L was elevated at $24 \mathrm{~h}$ postheading compared with pre-heading and $0 \mathrm{~h}$ post-heading time points, but the kicking (control) group remained static across all time points. The heading group's NF-L level at $24 \mathrm{~h}$ post-heading was higher than that of the kicking (control) group. Data are presented as the mean \pm SEM. NF-L = neurofilament light; SEM = standard error of the mean. The figure is reproduced from Wirsching et al. ${ }^{14}$ with permission from Mary Ann Liebert, Inc., New Rochelle, NY. Please click here to view a larger version of this figure.

\section{Discussion}

While contact sports like American football might appear to be driving the need for a concise research model to study subconcussive impacts, other sports like soccer may account for the dominant share of subconcussive exposure around the globe as approximately 265 million people participate in what is perhaps the world's most popular sport ${ }^{19}$. However, while the majority of the suspected long-term neurodegenerative effects of subconcussion have been autopsied in American football players, the resemblance of football head impacts to soccer headers is surprisingly large. For instance, 10 bouts of soccer heading in the current study induced approximately $300 \mathrm{~g}$ and $35 \mathrm{krad} / \mathrm{s}^{2}$, which were near identical to previous reports using similar subconcussion models ${ }^{20,21}$. These impact kinematics were comparable to the hits observed in American football, where the average college football player incurs 7.0-9.4 hits during practice and 25 hits during games, with a mean peak linear acceleration per hit of 28.8-32.0 $\mathrm{g}^{22,23,24,25}$. Furthermore, other contact sports, such as ice hockey, have displayed similar or greater peak linear head accelerations than these results. For example, a recent study examining both male and female collegiate ice hockey athletes revealed that, over the course of a season, these athletes would register peak linear accelerations of $41.6 \mathrm{~g}(36.6-49.5 \mathrm{~g})$ and $40.8 \mathrm{~g}(36.5-49.9 \mathrm{~g})$ for males and females, respectively (median [IQR]) ${ }^{26}$. Additionally, the head impact telemetry system that was utilized in this ice hockey study revealed that nearly one-third of males (28.0\% [21.2\%-33.5\%]) and females (29.3\% [24.8\%-32.1\%]) (median [IQR]) were inflicted on the front of the head ${ }^{26}$. These results directly parallel to the head impacts induced by using the SSHM, which indicates the vast clinical utility of the current methodology.

There is, however, one limitation that should be addressed when attempting to extrapolate soccer and American football head impacts to other contact sports like ice hockey. Head impacts in ice hockey are not routine drills and are penalized, and they usually are the result of deliberate contact with another player. In fact, subconcussive head impact incidence for collegiate ice hockey athletes is known to be substantially low: 1.3 $(1.0-1.7)$ per practice and $6.3(3.5-9.0)$ per game for males and $0.9(0.6-1.0)$ per practice and $3.7(2.5-4.9)$ per game for females ${ }^{26}$.

The present study is not the first to explore the expression of NF-L following head trauma; however, it is the first to isolate head trauma as the cause for NF-L expression outside the central nervous system. Oliver et al. assessed for serum NF-L expression within American football athletes at eight different time points over the course of a season ( 6 months $)^{17}$. Players were categorized into two groups, starters and nonstarters, with an assumption that starter athletes will be exposed to larger quantities of head hits, whereas nonstarters will incur lower quantities of head hits than the starters. The authors identified significant increases in serum NF-L expressions in the starter group over time across the season ${ }^{17}$. Conversely, the nonstarter group remained consistent throughout the season. Similarly, Shahim et al. analyzed serum NF$L$ levels in boxers following a subacute phase of subconcussive head trauma (7-10 days prior) ${ }^{18}$. In their analysis, boxers were categorized into two groups (mild vs. severe head impact groups) based on an anecdotal threshold of $<16 \mathrm{vs.} \geq 16$ head hits during a boxing match, respectively. The serum expression of NF-L was significantly elevated in the severe head impact group when compared to the mild impact group, suggesting a dose-dependent response to head impact and biomarker expression ${ }^{18}$. While the presented literature elicits strong reasoning for NF-L as a marker of axonal injury, neither study exhibits robust control of confounding variables seen throughout sport (thermogenesis, hydration status, body contacts, head kinematics, etc.). This gap in methodology underpins the need for the SSHM. By using the SSHM, Wirsching et al. $^{14}$ and Wallace et al. ${ }^{27}$ were able to control aforementioned confounding variables, coupled with the monitoring location, magnitude, and quantity of head impacts, and study the head impact dose-response profile of NF-L levels in the blood. Therefore, the SSHM provides safe and standardized means to study the effect of subconcussive head impacts and validate clinical findings.

The SSHM shows promise as being a relevant, reproducible, and leading method of isolating and examining the effect of subconcussive head impacts irrespective of sport; however, there are a few limitations to consider when adopting this method. First, the SSHM has a strong ability 
to control for environmental covariates (previously described); however, this means that a temperature-controlled indoor facility is required. Second, in an effort to ensure safe and controlled head contacts, we set participant exclusion criteria to a minimum of 5 years of soccer heading experience. This cutoff was used to eliminate potential injurious head contacts due to novice heading technique. Finally, this protocol requires specific equipment and multiple researchers to conduct, which may not be accessible unanimously.

The SSHM provides an invaluable modality for researchers to confidently validate results found in field studies. This confidence stems from the ability of the SSHM to control for both internal and external confounding factors such as impact location and quantity, body damage, exercise effect, and perspiration, to mention a few. Furthermore, because the SSHM has shown similar head impact magnitudes as other sports like ice hockey and American football, the claim can be made that this methodology bears vast clinical relevance not only to soccer athletes but also to American football, ice hockey, rugby, and boxing athletes. Lastly, because the SSHM does not segregate populations, as do some unisex sports like American football, all genders and ethnicities can be studied.

\section{Disclosures}

The authors have nothing to disclose.

\section{Acknowledgments}

The authors would like to acknowledge Ms. Angela Wirsching, who was a key contributor to the research we cite in the representative results section.

\section{References}

1. Goldstein, L.E. et al. Chronic traumatic encephalopathy in blast-exposed military veterans and a blast neurotrauma mouse model. Science Translational Medicine. 4 (134), 134ra60 (2012).

2. Kraus, M.F. et al. White matter integrity and cognition in chronic traumatic brain injury: a diffusion tensor imaging study. Brain: $A$ Journal of Neurology. 130 (Pt 10), 2508-2519 (2007).

3. McKee, A.C. et al. The spectrum of disease in chronic traumatic encephalopathy. Brain: A Journal of Neurology. 136 (Pt 1), $43-64$ (2013).

4. Mez, J. et al. Clinicopathological Evaluation of Chronic Traumatic Encephalopathy in Players of American Football. JAMA. 318 (4), $360-370$ (2017).

5. Tagge, C.A. et al. Concussion, microvascular injury, and early tauopathy in young athletes after impact head injury and an impact concussion mouse model. Brain: A Journal of Neurology. 141 (2), 422-458 (2018).

6. Bailes, J.E., Petraglia, A.L., Omalu, B.I., Nauman, E., Talavage, T. Role of subconcussion in repetitive mild traumatic brain injury. Journal of Neurosurgery. 119 (5), 1235-1245 (2013).

7. Estimated probability of competing in college athletics. NCAA.org - The Official Site of the NCAA. http://www.ncaa.org/about/resources/ research/estimated-probability-competing-college-athletics. Accessed September 20, 2018 (2018).

8. Schnebel, B., Gwin, J.T., Anderson, S., Gatlin, R. In vivo study of head impacts in football: a comparison of National Collegiate Athletic Association Division I versus high school impacts. Neurosurgery. 60 (3), 490-496 (2007).

9. Guskiewicz, K.M. et al. Measurement of head impacts in collegiate football players: relationship between head impact biomechanics and acute clinical outcome after concussion. Neurosurgery. 61 (6), 1244-1253 (2007).

10. Alfonsi, S. Combat veterans coming home with CTE. CBS News. https://www.cbsnews.com/news/60-minutes-combat-veterans-cominghome-with-cte-brain-injury/ (2018).

11. Ward, J., Williams, J., Manchester, S. 111 N.F.L. Brains. All But One Had C.T.E. The New York Times. https://www.nytimes.com/ interactive/2017/07/25/sports/football/nfl-cte.html. Accessed September 20, 2018 (2017).

12. Ling, $\mathrm{H}$. et al. Mixed pathologies including chronic traumatic encephalopathy account for dementia in retired association football (soccer) players. Acta Neuropathologica. 133 (3), 337-352 (2017).

13. Kawata, K., Tierney, R., Phillips, J., Jeka, J.J. Effect of Repetitive Sub-concussive Head Impacts on Ocular Near Point of Convergence. International Journal of Sports Medicine. 37 (5), 405-410 (2016).

14. Wirsching, A., Chen, Z., Bevilacqua, Z.W., Huibregtse, M.E., Kawata, K. Association of Acute Increase in Plasma Neurofilament Light with Repetitive Subconcussive Head Impacts: A Pilot Randomized Control Trial. Journal of Neurotrauma. (2018).

15. Coon, S. Acute Effects of Sleep Deprivation on Ocular-Motor Function as Assessed by King-Devick Test Performance. Master's thesis Bloomington, Indiana Indiana University (2018).

16. Hwang, S., Ma, L., Kawata, K., Tierney, R., Jeka, J.J. Vestibular Dysfunction after Subconcussive Head Impact. Journal of Neurotrauma. 34 (1), 8-15 (2017).

17. Oliver, J.M. et al. Serum Neurofilament Light in American Football Athletes over the Course of a Season. Journal of Neurotrauma. 33 (19), 1784-1789 (2016).

18. Shahim, P., Zetterberg, H., Tegner, Y., Blennow, K. Serum neurofilament light as a biomarker for mild traumatic brain injury in contact sports. Neurology. 88 (19), 1788-1794 (2017).

19. FIFA Communications Division, Information Services. FIFA Big Count 2006: 270 million people active in football. https://www.fifa.com/mm/ document/fifafacts/bcoffsurv/bigcount.statspackage_7024.pdf. Accessed April 9, 2018 (2007).

20. Dorminy, M. et al. Effect of soccer heading ball speed on S100B, sideline concussion assessments and head impact kinematics. Brain Injury. 1-7 (2015).

21. Bretzin, A.C., Mansell, J.L., Tierney, R.T., McDevitt, J.K. Sex Differences in Anthropometrics and Heading Kinematics Among Division I Soccer Athletes. Sports Health. 9 (2), 168-173 (2017).

22. Crisco, J.J. et al. Frequency and location of head impact exposures in individual collegiate football players. Journal of Athletic Training. 45 (6), 549-559 (2010).

23. Duma, S.M. et al. Analysis of real-time head accelerations in collegiate football players. Clinical Journal of Sport Medicine. 15 (1), 3-8 (2005). 
24. Reynolds, B.B. et al. Practice type effects on head impact in collegiate football. Journal of Neurosurgery. 1-10 (2015).

25. Kawata, K. et al. Association of Football Subconcussive Head Impacts With Ocular Near Point of Convergence. JAMA Ophthalmology. 134 (7), 763-769 (2016).

26. Wilcox, B.J. et al. Head impact exposure in male and female collegiate ice hockey players. Journal of Biomechanics. 47 (1), 109-114 (2014).

27. Wallace, C. et al. Heading in soccer increases serum neurofilament light protein and SCAT3 symptom metrics. BMJ Open Sport \& Exercise Medicine. 4 (1), e000433 (2018). 